\title{
Motor Restlessness
}

National Cancer Institute

\section{Source}

National Cancer Institute. Motor Restlessness. NCI Thesaurus. Code C117168.

An inability to be still and/or the need to move. 